elevated HDAC9 expression and to a decreased Foxp3 acetylation. Lower acetylated Foxp3 determines an instable phenotype of GARP-deficient Tregs and their failure to proper regulate an immune reaction.

Acknowledgements This work supported by DFG grants SK59/ 09-1 and Schu1683/10-1, and by BMBF Projekt Arthromark 01Ec1401B.

Disclosure of interest None declared

\section{P089 CHARACTERISATION OF CHEMOKINE RECEPTORS AND MIGRATION OF REGULATORY B CELLS IN PATIENTS WITH RHEUMATOID ARTHRITIS AND IN HEALTHY DONORS}

${ }^{1,2,3} \mathrm{R}$ Audo* ${ }^{1,2,3} \mathrm{~K}$ Schreiber, ${ }^{1,2,3}$ B Combe, ${ }^{1,2,3} \mathrm{~J}$ Morel, ${ }^{1,2,3} \mathrm{C}$ Daien. ${ }^{1} / \mathrm{GMM}$ UMR5535; ${ }^{2}$ Lapeyronie Teaching Hospital; ${ }^{3}$ Montpellier University, Montpellier, France

\subsection{6/annrheumdis-2018-EWRR2018.105}

Introduction B cells are pathogenic players in the development of rheumatoid arthritis (RA). More recently, it has been shown that $\mathrm{B}$ cells can also have regulatory functions, mainly through the secretion of interleukin-10 (IL-10). We found a decrease of IL10 +B (B10) cells in patients with early Rheumatoid Arthritis (RA) and B10 were inversely correlated with disease activity (DAS28), inflammation and rheumatoid factor, suggesting a key role at the initial phase of RA. Chemokines and chemokine receptors (CR) orchestrate migration of immune cells in physiological and pathological conditions. We hypothesise that CR could be involved in B10 functions and characterisation.

Objectives We aim to assess expression of CR on B10 and to understand their migration into the joint.

Methods Peripheral blood mononuclear cells (PBMCs) from 10 RA patients and 10 controls (HC) were activated 24 hours with $\mathrm{CpG}$ to generate B10 cells. We compared expression of several CR between B10 cells and IL- $10^{\text {neg }}$ B cells by FACS analysis. For functional test, B10 cells and IL-10 ${ }^{\text {neg }} \mathrm{B}$ were sorted by using secretion assay (Miltenyi) and the ability to migrate in response to CCL21, CCL22, CXCL11, CXCL12 or CXCL13 was evaluated by using migration assay in $5 \mu \mathrm{M}$ Transwell chambers. The presence of B10 cells in RA synovial tissue was evaluated by immunohistochemistry.

Results We found that B10 cells from HC differentially expressed several CR than IL- $10^{\text {neg }} \mathrm{B}$ cells, There were a strong decrease of CXCR5 and CXCR7 expression, and a strong increase of CCR4 expression in B10 cells $(p<0.001)$ compared to IL- $10^{\text {neg }}$ B cells. Functional impact of differential expression was tested by migration assay. Among chemokines tested,CXCL13, CXCR5-ligand, attracted more B10 cells than IL-10 ${ }^{\text {neg }} B$ cells $(n=7)$. More importantly, our results suggest that CR receptor profile on B10 cells is different between HC and RA $(n=10)$. Preliminary results showed that only a few B10 can be found in RA synovial tissue among B cells. One can hypothesised that the different expression profile of CR on B10 cells of RA patients might explain a defect in joint migration, and thus promoting uncontrolled inflammation.

Conclusions B10 cells have a specific CR profil compared to IL- $10^{\text {neg }} B$ cells. The differential expression of CR on RA B10 compared to HC might explain a defect in joint migration. Understanding of B10 migration is an important issue and could be used in the future to drive regulatory B cells into the joints as a new therapeutic approach for RA.
Disclosure of interest None declared

\section{P090 DIFFERENCE BETWEEN PALINDROMIC RHEUMATISM AND RHEUMATOID ARTHRITIS AT THE LEVEL OF GENE EXPRESSION}

S Ajaib*, A Droop, K Mankia, P Emery, F Ponchel. Leeds Institute of Rheumatic and Musculoskeletal Medicine, University of Leeds, Leeds, UK

\subsection{6/annrheumdis-2018-EWRR2018.106}

Introduction Palindromic rheumatism (PR) is characterised by recurrent, episodic attacks of articular inflammation, which resolve completely without residual joint damage. Whether PR should be considered as a prodrome of rheumatoid arthritis (RA) or as a distinct syndrome remains unclear, over 70 years since first described. ${ }^{1}$

Objectives This project investigates whether the pathogenesis of PR and RA is similar by comparing gene expression profiles from PBMCs in PR, healthy control (HC), and early RA patients.

Methods PBMCs were obtained from drug naïve PR patients during palindromic attack (flare, $\mathrm{n}=10$ ) and between attacks (non-flare, $\mathrm{n}=12$ ). 12 age/sex matched, drug nave RA patients were selected from the RA-MAP project along with $12 \mathrm{HC}$. The Illumina Human-HT-12/v4 Expression BeadChip microarray and a standard data analysis workflow, were used to establish a list of differentially expressed genes (DEGs). The STRING database was used to characterise functional associations of the top DEGs between groups. ${ }^{2}$ Only the highest confidence $(0.900)$ interactions were selected based on prior information from text-mining, experiments and databases.

Results Comparing RA and HC, STRING analysis revealed 112 interactions (from 378 DEGs); including expected associations with genes such as TNF, AP1 (JUN/FOS), chemokines/ receptors and a node including several Interferon responsive genes. Between PR non-flare and HC, 185 interactions (from 458 DEGs) were observed, featuring numerous endoplasmic reticulum and ribosomal proteins, in addition to high IL1beta/high IL-1R1, lower IL-10 and higher SOCS3. Interestingly, lower Ubiquitin $\mathrm{C}$ (UBC) was found to be a prominent feature of many of the identified interactions. Comparing inflammation between RA and PR flare, 176 interactions (from 476 DEGs) included an enhanced endoplasmic/ribosomal signature, lower ubiquitination but higher sumolation and other stress-associated proteins. SCOC3, was no longer present at the top of the DEGs list when both conditions were inflammatory. The IFN signature identified in RA was still highlighted in the associations. Finally, comparing PR non-flare and flare (10 matched pairs), 125 interactions (from 434 DEGs) were observed, primarily from pathways related again to stress, ubiquitination and DNA/RNA processing.

Conclusions Although preliminary, our findings suggest there are distinct gene expression programmes used in PR and RA, and that PR pathogenesis may involve several stress response mechanisms, which are not clearly associated with top DEGs in RA pathogenesis.

\section{REFERENCES}

1. Hench P, Rosenberg EF. Palindromic rheumatism. A 'new' oft recurring disease of joints (arthritis, periarthritis, para-arthritis) apparently producing no articular residues. Report of thirty-four cases; its relation to angio-neural arthrosis', 'allergic rheumatism' and rheumatoid arthritis. Arch Int Med 1944;73:293-321.

2. Szklarczyk D, Franceschini A, Wyder S, Forslund K, Heller D, Huerta-Cepas J, Simonovic $M$, Roth $A$, Santos $A$, Tsafou KP, Kuhn M, Bork $P$, Jensen LJ, von 